\title{
The Position of Clinical Pharmacists in Delivering Advanced Pharmacy Practice Education and Services
}

\author{
Minyahil Alebachew Woldu ${ }^{1 *}$, Alemseged Beyene Berha ${ }^{2}$, Demisew Berihun Haile ${ }^{3}$, Tamrat Assefa \\ Tadesse $^{4}$, Belete Ayalneh Worku ${ }^{5}$, Mamo Fayissa Senbeta ${ }^{6}$, Melaku Tileku Tamiru ${ }^{7}$
}

1-6Department of Pharmacology and Clinical Pharmacy, School of Pharmacy, College of Health Sciences, Addis Ababa University, Addis Ababa; Ethiopia

${ }^{7}$ Department of pharmacology and clinical pharmacy, school of pharmacy, college of health sciences, Addis Ababa University, Clinical pharmacy post graduate program PGY1, Addis Ababa; Ethiopia

\begin{abstract}
The pharmacist's role in health systems continues to evolve from a product focus to a patient-centered care model ensuring the safe and effective use of medications in all practice settings. The scope of pharmacy practice now includes patient-centred care with all the cognitive functions of counselling, providing drug information and monitoring drug therapy, as well as technical aspects of pharmaceutical services, including medicines supply management. Clinical Pharmacy consist of all the services accomplished by pharmacists practicing in hospitals, community pharmacies, nursing homes, home-based care services, clinics and any other setting wherever medicines are prescribed and used. Advanced pharmacy practice should be handled and delivered by facilities where high standards of pharmaceutical care and instruction are available and preceptors and educators should be actively engaged in the delivery of high-quality pharmaceutical care, and spend a majority of their time providing pharmaceutical care in their facility.
\end{abstract}

Key words: Pharmacy Practice, Clinical pharmacy, Advanced Pharmacy Practice.

\section{INTRODUCTION}

\section{Background: The Paradigm Shift in Pharmacy Services}

The pharmacist's role in health systems continues to evolve from a product focus to a patient-centered care model ensuring the safe and effective use of medications in all practice settings. As pharmacy practice evolves, the education and postgraduate training of pharmacists must ensure that pharmacists are adequately prepared to assume advanced practice roles in direct patient care. ${ }^{1,2}$ The introduction of experiential learning in pharmacy practice curricula is therefore very mandatory as it provides the students with practical experience in various aspects of the profession of pharmacy. ${ }^{3}$

Current pharmacy practice is considerably more diverse than what has been previously reported in terms of scope of practice and practice setting. ${ }^{4}$ It is therefore timely to consider professional pharmacy practice and the way in which we can define and progress the well accepted concept of 'advanced pharmacy practice'. ${ }^{5}$ A pharmacist preceptor (PharmD and clinical pharmacists) directs the majority of practice experiences. The objective of the current short communication is to make readers familiar with the position of clinical pharmacists in provision of pharmacy practice courses and services and to show them that these two names are two faces of same coin and hence can be used interchangeably unless and otherwise specified.

\section{Scope of Practice of Clinical Pharmacy}

Education, training, experience, and awareness of practice standards and trends help create the practice model vision. ${ }^{1}$ The scope of pharmacy practice now includes patient-centred care with all the cognitive functions of counselling, pro-
DOI: 10.5530/ijopp.9.3.9

Address for correspondence:

Minyahil Alebachew Woldu, Department of pharmacology and clinical pharmacy, School of pharmacy, College of health sciences, Addis Ababa University, P.O. Box 9086, Addis Ababa, ETHIOPIA.

Phone no: +251-912-648527

E-mail: minwoldu@gmail.com

(n)


viding drug information and monitoring drug therapy, as well as technical aspects of pharmaceutical services, including medicines supply management. ${ }^{6}$ Clinical pharmacy services to support the national medicines policy includes: access, equity and continuum of care, decision support tools, drug use evaluation, patient information and education programs, clinical risk management, training and education and research. ${ }^{7}$

Clinical pharmacists should be involved in activities directed to individual patients and more broadly to support the objectives of the national medicines policy, especially its quality use of medicines (QUM) arm. Procedures for clinical pharmacy services for individual patients include Medication Action Plan Interpretation of Patient-Specific Data, Identification of Clinical Problems, Establishment of Therapeutic Goals, Evaluation of Therapeutic Options, Individualisation of Therapy, and Monitoring of Patient Outcomes]. ${ }^{8}$

Clinical pharmacy practice is the practice of pharmacy as part of a multidisciplinary healthcare team directed at achieving QUM. This may include participation in the management of individual patients; application of the best available evidence in daily clinical practice; and contribution of clinical knowledge and skills to the healthcare team. ${ }^{8}$ The primary focus of community pharmacy as component of pharmacy practice is to provide professional care to patients, supported by safe and effective medication management and medication information, comprehensive warfarin counselling, smoking cessation counselling, MUR-medicine use reviews, free emergency contraception counselling and dispensing, flu vaccination campaigns, waste management and DUMP (Dispose Unwanted Medicines Programme) and medication counselling for patients initiated on medicines for mild to moderate depression in which all require an in-depth clinical pharmacy knowledge. ${ }^{7}$

\section{Pharmacy Practice versus Clinical Pharmacy}

The "Practice of Pharmacy" means the interpretation, evaluation, and implementation of Medical Orders; the Dispensing of Prescription Drug Orders; participation in Drug and Device selection; Drug Administration; Drug Regimen Review; the Practice of Telepharmacy within and across state lines; Drug or Drug-related research; the provision of Patient Counselling; the provision of those acts or services necessary to provide Pharmacist Care in all areas of patient care, including Primary Care and Collaborative Pharmacy Practice; and the responsibility for Compounding and Labelling of Drugs and Devices (except Labelling by a Manufacturer, Repackaging, or Distributor of Non-Prescription Drugs and commercially packaged Legend Drugs and Devices), proper and safe storage of Drugs and Devices, and maintenance of required records. The practice of pharmacy also includes continually optimizing patient safety and quality of services through effective use of emerging technologies and competency-based training., ${ }^{9}, 10$ Clinical Pharmacy is a health science discipline in which pharmacists provide patient care that optimizes medication therapy and promotes health, wellness, and disease prevention. To achieve desired therapeutic goals, the clinical pharmacist applies evidence-based therapeutic guidelines, evolving sciences, emerging technologies, and relevant legal, ethical, social, cultural, economic, and professional principles. ${ }^{11}$ The clinical pharmacist is trained in clinical pharmacy practice and comprehensive medication management to include, but not limited to clinical pharmacokinetics, therapeutics, and clinical pharmacology. ${ }^{12}$

Clinical Pharmacy consist of all the services accomplished by pharmacists practicing in hospitals, community pharmacies, nursing homes, home-based care services, clinics and any other setting wherever medicines are prescribed and used. The term "clinical" does not necessarily suggest an action implemented only in a hospital settings. ${ }^{13} \mathrm{~A}$ clinical pharmacist has the unique mix of knowledge, skills, and abilities in addition to education, training and experience to function under a scope of practice. Furthermore, the clinical pharmacist has a current unrestricted pharmacist license and is in good standing with the pharmacist's licensing body. ${ }^{12}$

The University Health System Consortium (CHC) work force concluded that institutions need a practice model to support basic medication management services on a consistent basis for all patients and specialized services for specific patients depending on their clinical situations. Technology may help achieve this goal, but a well-trained workforce and an appropriate model design are critical for success. ${ }^{1}$

\section{The Global view of Pharmacy Practice and Clinical pharmacy}

In U.S. and India, pharmacy practice mean a postgraduate course designed to make students familiar with patient care rotations, institutional practice, community practice and ambulatory care practices. ${ }^{14,15}$ According to Introductory Pharmacy Practice Experience (IPPE) and Advanced Pharmacy Practice Experience (APPE) Manual of the University of Georgia, the purpose of the experiential training portion of the curriculum is to provide pharmacy students with practical experience in various aspects of the profession of pharmacy. A pharmacist preceptor (PharmD and clinical pharmacists) directs the majority of practice experiences. APPEs are designed to provide students the opportunity to focus on clinical aspects of pharmacy practice. These expe- 
riences usually involve direct patient care in a specific clinical area (e.g., cardiology, oncology, pediatrics, etc.). However, some experiences may not have direct patient care (e.g., drug information, pharmaceutical industry, research, etc.). ${ }^{16}$

According to Queens University Belfast, advance pharmacy practice is a 12 module over 54 month period course and aims to make pharmacists to be independent prescribers in health system facilities. These modules are focusing in hospital pharmacy, clinical medicine, disease management, advanced therapeutics, development of leadership skills; drug distribution and control systems; ethical practices and research. ${ }^{17,18}$

According to University of Houston, college of pharmacy, students taking advanced pharmacy practice shall able to perform the functions of a pharmacist in a hospital setting including dispensing Function: in-patient area, manufacturing, compounding, repackaging, controlled substances, and investigational drugs. Management Function: operations, purchasing, inventory control. Educational Function: drug information, healthcare professional education, patient education and Pharmaceutical Care Function: monitoring, evaluating drug therapy, emergency pharmacy services, communication. ${ }^{19}$

\section{The Situations in Ethiopia}

In Ethiopia-Jimma University, took the lead in clinical pharmacy using the advantages of its unique experiential learning practice sites and its community-based approach to learning, which allows students to train in the university's teaching hospital and local training health centres. The Clinical Pharmacy and Pharmacy Practice (CPPP) course team is lead by clinical pharmacists and provides more than $40 \%$ of the major courses required for BPharm program and owns all courses for the MSc program in Clinical pharmacy. ${ }^{20,21}$

At Addis Ababa University (AAU), the Advanced Pharmacy Practice course of the MSc in Pharmacy practice program has been designed for students to make students familiar with the advanced pharmacy practice services in hospitals and community pharmacies. Under the hospital pharmacy chapter the following areas are targeted: overview of organization and structure, basic and specialized hospital pharmacy services; hospital drug policy: drug and therapeutics committee, formulary and guidelines, research \& ethics committee, infection control committee, patient safety/medication safety. hospital pharmacy management: staff, finance, policy and planning, infrastructural requirements, drug distribution system, pharmaceutical care, drug therapy needs of a patient, drug therapy problems, pharmaceutical care process. Developing a clinical approach in clinical pharmacy practice: Clinical information: clinical notes, records and charts, pharmaceutical interventions, understanding clinical laboratory tests, screening patients for medication problems, reviewing medication histories, ethics and confidentiality. Communicating information: developing questioning, explaining and listening skills, non-verbal communication, undertaking patient consultations. Medicines information: searching for and evaluating medicines information, Evidence-based Medicine and Medication therapy management in which all require an in-depth clinical pharmacy knowledge. ${ }^{22}$

Under the community pharmacy chapter also the following points were given focus: Overview of organization, administration, drug supply management; dispensing, prescription and OTC medicines, responding to symptoms, dispensing of herbal/alternative medicines, medication therapy management that has been addressed in the earlier international experiences can be again addressed by clinical pharmacists. ${ }^{22}$

\section{Qualification requirement for Clinical Pharmacy Practice Course}

According to ACCP commentary, Postgraduate Training - PGY1 Residency Training should have to be handled by all full-time clinical pharmacy practice faculty, as well as all adjunct clinical faculty with direct patient care responsibilities and all actively precepting students, complete a PGY1 residency or possess equivalent experience. ${ }^{23}$ It is essential that advanced pharmacy course should be provided in facilities where high standards of pharmaceutical care and instruction are available. Therefore, Patient Care Advanced Practice Experience preceptors and sites must meet certain qualifications criteria. The clinical preceptor should be a clinical scientist with expertise in the area of pharmD, clinical pharmacy/pharmacy practice, which may be exemplified by: receiver of fellowship training, a graduate degree, and/or equivalent experiences ${ }^{24}$ and be actively engaged in the delivery of high-quality pharmaceutical care, and spend a majority of their time providing pharmaceutical care in their facility but also will commit sufficient time to education. ${ }^{19}$

\section{CONCLUSION}

Clinical pharmacy practice has developed internationally to expand the role of a pharmacist well beyond the traditional roles of compounding and supplying drugs to roles more directly in caring for patients and providing medication consultation to staff. This area of practice is at its infant age in Ethiopia. Advanced pharmacy practice should be handled and delivered by facilities where high standards of pharmaceutical care and instruction are available and preceptors and educators should be actively engaged in the delivery of high-quality pharma- 
ceutical care, and spend a majority of their time providing pharmaceutical care in their facility.

\section{ACKNOWLEDGEMENT}

Addis Ababa University, college of Health sciences and school of pharmacy

\section{CONFLICT OF INTEREST}

The authors would like to announce that there is no confilict of interest regarding this work

\section{ABBREVIATION USED}

AAU: Addis Ababa University

ACCP: American college of Clinical pharmacy

APPE: Advanced Pharmacy Practice Experience

CPPP: Clinical Pharmacy and Pharmacy Practice

DUMP: Dispose Unwanted Medicines Programme

IPPE: Introductory Pharmacy Practice Experience

JU: Jimma University

MSc: Master of Sciences

MUR: Medicine Use Reviews

PGY1: Postgraduate year 1

PharmD: Doctor of Pharmacy

QOM: Quality use of Medicines

\section{REFERENCES}

1. UHC: Pharmacy Practice Model for Academic Medical Centers. In. Edited by Consortium UH. Oak Brook; 2010.

2. ACCP: Desired Professional Development Pathways for Clinical Pharmacists. In. Edited by Nancy Yunker, Stacy S. Shord, Terry L. Schwinghammer, Melissa Badowski, Julie Banderas, Michael E. Burton, Christopher A. Chapleau, Jason C. Gallagher, Gregory Matsuura, Sara E. Parli a. Lenexa: American College of Clinical Pharmacy; 2012.

3. Mebratu Legesse, Gebrehiwot Teklay, Derbew Fikadu, Birhanu Demeke: Clerkship guideline for undergraduate pharmacy students. In. Mekelle: Mekelle University College of Health Sciences Department of Pharmacy; 2012.
4. RADM Scott Giberson, CDR Sherri Yoder, CDR Michael P. Lee: Improving Patient and Health System Outcomes through Advanced Pharmacy Practice. In: A Report to the US Surgeon General 2011

5. RPSGB: Recognition of advanced pharmacy practice in australia. In: Competency Standards Review Steering Committee. 2010.

6. WHO, FIP: Developing pharmacy practice: A focus on patient care. In. Edited by Standards DoMPa. Geneva, Switzerland; 2006.

7. Jenny West, Janet Amey, Cath Knapton, Illing. S: Clinical pharmacy in general practice. Midlands Health Network 2012.

8. SHPA: SHPA Standards of Practice for Clinical Pharmacy. J Pharm Pract Res. 2005;35(2):122-46. http://dx.doi.org/10.1002/j.2055-2335.2005.tb00322.x.

9. Council on Credentialing in Pharmacy: Scope of Contemporary Pharmacy Practice: Roles, Responsibilities, and Functions of Pharmacists and Pharmacy Technicians. In. Edited by Pharmacy TCoCi. Washington, DC: CCP; 2009.

10. Pharmacy; NBo, Pharmacy; NAoBo: Model State Pharmacy Act and Model Rules of the 3. In.; 2008.

11. ACCP:The Definition of Clinical Pharmacy. Pharmacotherapy. 2008;28(6):816-7. http://dx.doi.org/10.1592/phco.28.6.816; PMid:18503408.

12. Veterans Health Administration: Clinical pharmacy services. In. Edited by Affairs DoV. Washington; 2015.

13. Implementation of Advances and Challenges in Clinical Pharmacy. In: 3rd International Conference on Clinical Pharmacy. Atlanta, USA; 2015

14. Toronto Uo: Advanced Pharmacy Practice Experience. In. Edited by Toronto Uo; 2015.

15. WSOP: Advanced pharmacy practice experiences (appe) manual In.; 2015.

16. Pharmacy TUoGCo: Introductory Pharmacy Practice Experience (IPPE) and Advanced Pharmacy Practice Experience (APPE) Manual In.; 2014.

17. Belfast QU: MSc in Advanced Pharmacy Practice (with Independent Prescribing). In.; 2013.

18. TTUHSC SOP: Introduction to Pharmacy Practice. In. Edited by TTUHSC. Amarillo; 2012.

19. University of Houston: Advanced hospital pharmacy practice experience course description. In. Edited by Pharmacy UoHCo; 2014.

20. School of pharmacy, University J: Pharmacy course syllubus. In.; 2009.

21. Mekonnen AB, Yesuf EA, Odegard PS, SS. W: Implementing ward based clinical pharmacy services in an Ethiopian University Hospital. Pharmacy Practice. 2013; 11(1):51-7. http://dx.doi.org/10.4321/S1886-36552013000100009; PMid:24155850 PMCid:PMC3780502.

22. School of pharmacy, AAU: Pharmacy practice course syllubus. In.; 2010

23. Janet P. Engle, Brian L. Erstad, Douglas C. Anderson, Jr. MHB, Alexandre Chan, Amy R. Donaldson, Tracy M. Hagemann, Mary Beth O'Connell, Philip T. Rodgers, and ST et al: Minimum Qualifications for Clinical Pharmacy Practice Faculty. In. Edited by Pharmacy ACoC; 2013.

24. ACCP: Guidelines for Clinical Research Fellowship Training Programs. In: The ACCP Board of Regents. 2004. 